\title{
EXISTE RELAÇÃO ENTRE FRAGILIDADE E SONO DE IDOSOS NA COMUNIDADE?
}

Sonia Gonçalves da Mota; Universidade Federal de São Carlos; soniagmota_47@hotmail.com

Marcela Naiara Graciani Fumagale Macedo; Universidade Federal de São Carlos; marcelagracianivest@hotmail.com

Marcos Soares de Arruda; Universidade Federal de São Carlos, marcossarruda@gmail.com Tabatta Renata Pereira de Brito; Universidade Federal de Alfenas; tabatta_renata@hotmail.com Élen dos Santos Alves; Universidade Federal de São Carlos; elendutra23@gmail.com Keika Inouye; Universidade Federal de São Carlos; keikain@ufscar.br Ariene Angelini dos Santos-Orlandi; Universidade Federal de São Carlos; arieneangelini@yahoo.com.br

\section{RESUMO}

Introdução: As queixas relacionadas ao sono e a síndrome da fragilidade são frequentes entre idosos. Ambas condições podem influenciar negativamente a saúde e o bem estar dos indivíduos acometidos. Objetivo: Identificar associação entre sono e fragilidade de idosos no contexto de alta vulnerabilidade social. Métodos: A amostra $(n=81)$ foi composta por indivíduos com 60 anos ou mais, cadastrados em uma Unidade de Saúde da Família no município de São Carlos, SP. Foram utilizados os seguintes instrumentos: Questionário para Caracterização do Idoso, Índice de Qualidade do Sono de Pittsburgh e Fenótipo de Fragilidade proposto por Fried. Foram realizadas análises estatísticas descritivas e comparativas (Exato de Fisher e $\mathrm{X}^{2}$ de Pearson). Resultados: a maioria dos idosos era do sexo feminino $(51,8 \%)$ e estava inserida na faixa etária de 60 a 69 anos $(65,4 \%)$. Houve predomínio de idosos pré-frágeis $(61,7 \%)$, seguidos de idosos frágeis $(33,3 \%)$ e robustos (5,0\%). Quanto ao sono, 50,6\% apresentaram sono de má qualidade e 16,1\% distúrbios do sono. Dos idosos robustos e pré-frágeis, $50,0 \%$ pontuaram para má qualidade do sono e 9,3\% para distúrbios do sono. Dos idosos frágeis, 51,9\% pontuaram para má qualidade do sono e 29,6\% para distúrbios do sono. Houve diferença entre os grupos $(p=0,026)$. Conclusão: Conclui-se que existe associação entre fragilidade e sono de idosos na comunidade. Idosos frágeis apresentaram maior prevalência de sono de má qualidade e de distúrbios do sono quando comparados aos idosos pré frágeis e não frágeis.

Palavras-chave: Idoso Fragilizado; Sono; Enfermagem Geriátrica. 